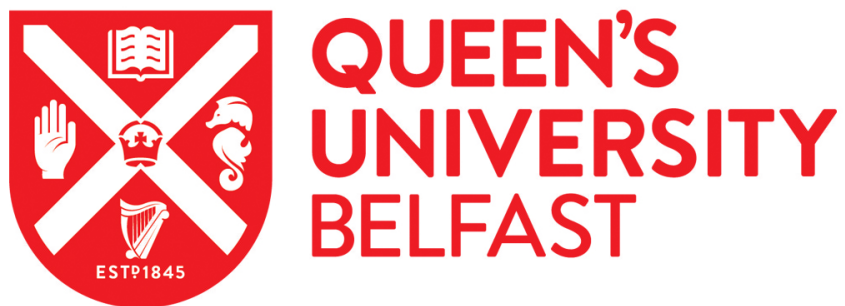

\section{The optimization of process analytical technology for the inline quantification of multiple drugs in fixed dose combinations during continuous processing}

Dadou, S. M., Tian, Y., Li, S., Jones, D. S., \& Andrews, G. P. (2020). The optimization of process analytical technology for the inline quantification of multiple drugs in fixed dose combinations during continuous processing. International Journal of Pharmaceutics, [120024]. https://doi.org/10.1016/j.ijpharm.2020.120024

Published in:

International Journal of Pharmaceutics

Document Version:

Peer reviewed version

Queen's University Belfast - Research Portal:

Link to publication record in Queen's University Belfast Research Portal

\section{Publisher rights}

Copyright 2020 Elsevier.

This manuscript is distributed under a Creative Commons Attribution-NonCommercial-NoDerivs License

(https://creativecommons.org/licenses/by-nc-nd/4.0/), which permits distribution and reproduction for non-commercial purposes, provided the author and source are cited

\section{General rights}

Copyright for the publications made accessible via the Queen's University Belfast Research Portal is retained by the author(s) and / or other copyright owners and it is a condition of accessing these publications that users recognise and abide by the legal requirements associated with these rights.

\section{Take down policy}

The Research Portal is Queen's institutional repository that provides access to Queen's research output. Every effort has been made to ensure that content in the Research Portal does not infringe any person's rights, or applicable UK laws. If you discover content in the Research Portal that you believe breaches copyright or violates any law, please contact openaccess@qub.ac.uk. 


\section{The Optimization of Process Analytical Technology for The Inline Quantification of Multiple Drugs in Fixed Dose Combinations during Continuous Processing}

Suha M. Dadou ${ }^{\mathrm{a}, \mathrm{b}}$, Yiwei Tian ${ }^{\mathrm{a}}$, Shu Li ${ }^{\mathrm{a}, \mathrm{b}}$, David S. Jones ${ }^{\mathrm{a}}$, Gavin P. Andrews ${ }^{\mathrm{a}, \mathrm{b}}$

a Pharmaceutical Engineering Group, School of Pharmacy, Queen's University Belfast, Belfast BT9 7BL, United Kingdom

${ }^{\mathrm{b}}$ China Medical University - Queen's University Belfast joint College (CQC) /

Pharmaceutical Engineering Group, School of Pharmacy, Queen's University Belfast, Belfast BT9 7BL, United Kingdom

* Corresponding author at: School of Pharmacy, 97 Lisburn Road, Belfast BT9 7BL, United Kingdom.

Email address: g.andrews@qub.ac.uk (G.P. Andrews)

Tel: +442890972646

Keywords:

Continuous manufacturing, hot melt extrusion, fixed dose combination, process analytical technology, inline Raman spectroscopy 


\section{ABSTRACT}

Complications associated with uncontrolled hypertension are considered the major cause of premature death worldwide. Fixed-dose combinations (FDCs) offer an alternative approach to polypharmacy with the aim to improve patient compliance. Process Analytical Technology (PAT) is gaining momentum as a non-invasive, predictive tool to control the quality of drugs during continuous processing. PAT offers real-time quality control that can be built into the production line. However, the vast majority of studies reported in the literature have focused on quantifying a single drug during continuous processing. The aim of this study was to develop non-destructive, predictive inline PAT tools allowing for the simultaneous quantification of two antihypertensive drugs, Hydrochlorothiazide (HCTZ) and Ramipril (RMP), during the continuous manufacture of FDCs. A calibration set composed of HCTZ and RMP at concentration ranges of 6.5 to 40 and $2.5-15(\% \mathrm{w} / \mathrm{w})$, respectively, were manufactured using hot melt extrusion. The extrudates were analysed during the process using inline Raman spectroscopy. Optimum wavenumber regions were observed at $200-400$ and $630-730 \mathrm{~cm}^{-1}$ for HCTZ, and 980-1100 $\mathrm{cm}^{-1}$ for RMP using principal component analysis. Partial least squares (PLS) regression was performed to establish the predictive calibration models. The PLS developed models showed excellent linearity $(\mathrm{R} 2=0.986$ and 0.974$)$, selectivity $(\mathrm{PC} 1=98.6 \%$ and $91.9 \%)$ and accuracy $(\mathrm{RMSEcv}=1.586$ and $0.645 \%)$ for HCTZ and RMP, respectively. Additionally, RMSEP values were reported as 1.237 and $1.007 \%$ for HCTZ and RMP, respectively, depicting good predictability for drug content in the validation set. The output of this study demonstrated that utilisation of the full potential of chemometrics, Raman spectroscopy can be used for the simultaneous inline quantification of multiple drugs in complex formulations. This facilitates the in-process quality control of FDCs and other multicomponent systems during continuous pharmaceutical production. 


\section{Introduction}

Hypertension is a prevalent global health issue. An estimated 1.4 billion people have hypertension globally (Kishore et al., 2018). This is due the fact that most hypertensive patients develop no signs; thus, are unaware of their health condition (World Health Organization, 2019). Furthermore, poor patient compliance due to long term, multiple dosage regimens is a major challenge that often leads to a compromised clinical efficiency and a magnification in the risk of complications associated with uncontrolled hypertension (Andrews et al., 2019b; Kishore et al., 2018). On average, 55\% of patients suffering from chronic health conditions who fail to adhere to their prescribed drug regimens are hypertensive patients (Abegaz et al., 2017).

The use of fixed-dose combinations (FDCs) is emerging as the standard of care for the management of chronic diseases. FDCs are drug dosage forms combining two or more active pharmaceutical ingredients (APIs) in a single dosage form (Oo and Sy, 2018). They offer a potent alternative to simplify complex dosing regimens (US Food and Drug Administration, 2014). FDCs have substantial advantages including reduced dosing frequency, improved medication adherence, and reduced medication costs (Andrews et al., 2019b; Hao et al., 2015). Therefore, FDCs were well recognised and highly endorsed by the European Medical Agency (EMA) for the management of hypertension (EMA, 2017). Guidance documents were released to regulate the strategies used for the development and production of FDC commercial products. As a result, FDCs have been produced by major pharmaceutical companies and used for a number of different therapeutic areas. More recently, FDCs of antihypertensive medications were officially added to WHO's Essential Medicines List (EML) (Salam et al., 2019). Combinations from different anti-hypertensive classes were endorsed based on worldwide availability and best available evidence. FDCs comprising angiotensin-converting enzyme inhibitors (mainly Ramipril) and thiazide diuretics (e.g., Hydrochlorothiazide) were 
the first to be reviewed and recognised by the WHO expert panel as they form one of the most commonly used and globally available anti-hypertensive combinations.

Despite the tremendous advantages FDCs offer, current manufacturing techniques and methods/tools used for quality control and assurance are rather complex. Therefore, novel technologies have been investigated during the last decade to assess their feasibility for the manufacture of FDCs. Hot melt extrusion (HME) has been extensively investigated for the production of pharmaceuticals during the last two decades (Tiwari et al., 2016; Wilson et al., 2012). Several studies have confirmed the potential of HME as an advanced technology platform for the manufacture of FDCs (Andrews et al., 2019b; Dierickx et al., 2012). Nevertheless, HME has not been widely implemented as a means of routine oral dose manufacture in the pharmaceutical industry. Major challenges include regulatory uncertainty towards such manufacturing platforms, and the need to reconsider the infrastructure of companies to accommodate the emerging view of advanced manufacturing technologies (Dadou et al., 2020b; Lee, 2017).

A Process Analytical Technology (PAT) regulatory framework was introduced by the Food and Drug Administration (FDA) as a quality control tool to facilitate the transition into continuous manufacturing platforms within the pharmaceutical industry (FDA, 2004). Implementing PAT to the production line enables the real-time release testing of materials during manufacturing by employing non-destructive technologies (Dadou et al., 2020b; Hisada et al., 2020). Thus, the quality of formulations and/or processes can be controlled during pharmaceutical process, such as drug quantification, water content and monitoring the solidstate of drugs (Koide et al., 2020; Nomura et al., 2020). However, the automation of quality control methods by integrating PAT to the process is still in its infancy. Rather, offline destructive analytical methods are still widely used as routine methods to assess/control the quality of end products and/or processes in the current conventional batch methods. 
In our previous work, we confirmed the feasibility of utilising HME as a continuous processing platform for the manufacture of FDCs for the management of chronic diseases (Andrews et al., 2019b; Kelleher et al., 2018). Additionally, we demonstrated the suitability of Raman spectroscopy for the inline quantification of Ramipril during processing by utilising the quality by design approach and PAT framework (Andrews et al., 2019a; Dadou et al., 2020b). In this work, a FDC composed of Hydrochlorothiazide (HCTZ) and Ramipril (RMP) was manufactured using HME for the management of hypertension. To date, work investigated in the scientific literature has focused on analysing a single drug/ingredient during continuous processing. The aim of this work was to demonstrate the feasibility of utilising inline Raman spectroscopy as a non-invasive, PAT tool for the simultaneous quantification of two distinct drug entities (HCTZ and RMP) in FDCs manufactured using HME.

\section{Materials and Methods}

\subsection{Materials}

Ramipril (RMP) was purchased from Kemprotec (Carnforth, England). Hydrochlorothiazide (HCTZ) was purchased from Alfa Aesar (Lancashire, England). Eudragit EPO (EPO) and Eudragit L 100 (L100) were obtained from Evonik Industries (Darmstadt, Germany). Triethyl citrate (TEC) was purchased from Lancaster synthesis Ltd (Morecambe, UK). All other chemicals were of analytical grade or equivalent and were used with no further treatment.

\subsection{Hot Melt Extrusion (HME)}

HME was performed using a co-rotating, fully intermeshing twin-screw extruder (Microlab, Rondol Technology Ltd, France). Physical mixtures of the drugs, polymers and plasticiser were mixed using a mortar and pestle. The ratio of EPO:L100 was kept constant at 1:1 in all formulations. TEC concentration was fixed at $7.5 \% \mathrm{w} / \mathrm{w}$. The polymers to drugs ratio was altered based on the content of drugs used as shown in Table 1. The premixed powders were then fed into the extruder using a twin-screw powder feeder (Rondol Technology Ltd, France) 
at feed rate of $32 \mathrm{rpm}$. Extrusion was carried out at a screw speed of $80 \mathrm{rpm}$ and a barrel temperature of $106{ }^{\circ} \mathrm{C}$. An in-house designed, $2 \mathrm{~mm}$ diameter die was fitted to the end of the barrel to accommodate the inline Raman probe. The extrudates produced were collected and stored in sealed bags for further analysis.

\subsection{Determination of Drug Content using High Performance Liquid Chromatography (HPLC)}

HPLC analysis was performed using an Agilent 1260 Infinity Series HPLC (Agilent Technologies, Cheadle, UK). A Kinetex ${ }^{\circledR}$ C18 column $(150 \mathrm{~mm} \times 4.6 \mathrm{~mm}$, phenomenex, Torrance, USA) was used as the stationary phase. $10 \mu \mathrm{L}$ of each sample was injected to the column using an auto-sampler. The mobile phase consisting of an aqueous solution of $0.1 \mathrm{M}$ sodium perchlorate adjusted to $\mathrm{pH} 2.5$ using phosphoric acid (mobile phase A), and acetonitrile (mobile phase B) was pumped to the system under gradient mode at varied flow rates between 0.8 and $1.5 \mathrm{~mL} / \mathrm{min}$. Detection was achieved using a UV detector at a wavelength of $210 \mathrm{~nm}$. The area under the peak of each drug was used to calculate its concentration in the sample. HPLC offline was used as the reference method of analysis to quantify RMP and HCTZ in the extrudates. Following extrusion, pelletised extrudates were placed, separately, in volumetric flasks and dissolved in the mobile phase to obtain a final concentration of the drugs within the range used to prepare the calibration curves. Aliquots were withdrawn from the prepared samples, filtered through hydrophilic PTFE syringe filters $(0.45 \mu \mathrm{m}$, Fisher Scientific Ireland Ltd., Dublin, Ireland) then transferred into the HPLC vials and analysed.

\subsection{Raman Spectroscopy Measurements}

A portable benchtop Raman Rxn1 spectrometer (Kaiser Optical Systems, Ann Arbor, MI, USA) equipped with an Invictus NIR diode laser was used to collect Raman Spectra. A high temperature and pressure immersion HME Kaiser Probe (RAMAN RXNTM Probe) was installed into the extruder die for inline measurements. All Raman spectra were recorded using a Kaiser Optical Systems at a laser power of $400 \mathrm{~mW}$ and wavelength of $785 \mathrm{~nm}$. Spectra were 
collected every 30 seconds during extrusion using a resolution of $2 \mathrm{~cm}^{-1}$ and a total exposure time of 20 seconds. Data collection was automated using iC Raman software (version 4.1, Mettler Toledo, US). Prior to performing inline measurements, Raman spectra were collected offline for raw materials individually, using the same Raman settings, to identify the characteristic Raman shifts and wavenumber regions for each component in the FDC.

\subsection{Principal Component Analysis (PCA)}

The analysis of the Raman spectra collected during extrusion was achieved using SIMCA software (version 15, Umetrics, Umeå, Sweden). To reduce random noise and baseline drift in the signal, particularly at high temperature, Standard Normalised Variate (SNV) or first derivative (1D) pre-processing spectral filters were considered. For both filters, Multiplicative Scatter Correction (MSC) and 15-point quadratic Savitzky-Golay (SG) were further applied to the spectra. Analyses were performed on different spectral regions to identify the regions and pre-processing filters that best represent/quantify each drug in the extrudates. Principal Component Analysis (PCA) was performed to identify the principle components that capture the variation in spectra collected for each drug during HME.

\subsection{The Development of Inline Raman based Calibration Models}

A calibration set composed of formulations C1 to C5 (Table 1) was used to develop the calibration models from inline Raman spectra. FDCs of HCTZ and RMP were manufactured at theoretical concentration range of 6.5 to $40(\% \mathrm{w} / \mathrm{w})$ and $2.5-15(\% \mathrm{w} / \mathrm{w})$, respectively, to cover a wide range of the therapeutic relevant doses available in commercial products. SIMCA software (version 15, Umetrics, Umeå, Sweden) was used to build the calibration models. Partial Least Square (PLS) calibration models were established by regressing the inline Raman spectra collected during HME from the calibration set, against the corresponding actual concentrations measured using offline HPLC. Separate PLS calibration models were established for HCTZ and RMP. 


\subsection{Validating and Evaluating PLS Calibration Models}

The software (SIMCA) built-in cross-validation tool was used for the internal validation of the model. For external validation, a validation set was manufactured using HME process conditions and Raman settings previously described (formulations V1- V3, Table 1). The actual concentrations of drugs in the extrudates were measured using offline HPLC and used for external validation. Validation terms including selectivity, linearity, accuracy and precision were assessed to meet the validation criteria required by existing guidelines (EMA, 2009; FDA, 2015). Parameters used to evaluate the PLS model performance were the coefficient of determination of $\left(\mathrm{R}^{2}\right)$ of calibration models, principal components (PC) of the loading plots, root mean squared error of estimation (RMSEE), root mean squared error of cross validation (RMSEcv) and root mean square error of prediction (RMSEP). On the other hand, relative standard deviation (RSD, \%) was calculated for the validation set and used to evaluate precision. In addition, the relative bias between the true value of each measurement (HPLC) and the corresponding value predicted using the PLS calibration models was estimated using Eq. (1) (Harting and Kleinebudde, 2018).

$$
\text { Relative bias }(\%)=\frac{\left(\hat{y}_{i}-y_{i}\right)}{y_{i}} \times 100
$$

where $y_{i}$ is the true value calculated by HPLC and $\hat{y}_{i}$ is the predicted value. 


\section{Results and Discussion}

This work follows on from previous publications by our group wherein Raman spectroscopy was successfully employed as a PAT tool for the inline quantification of Ramipril (RMP) during HME (Andrews et al., 2019a; Dadou et al., 2020b). This was achieved by optimising the quality attributes affecting RMP stability during HME with the aid of QbD framework. In addition, the quality of RMP was monitored during the process through a validated Raman based PAT tool. The outcome enabled the use of HME as a continuous processing platform for the manufacture of thermolabile drug products, e.g., RMP. Herein, we have investigated the feasibility of using inline Raman as a PAT tool for the simultaneous quantification of RMP and Hydrochlorothiazide (HCTZ) in fixed dose combinations (FDC). Formulation composition and process conditions previously optimised through the validated design space (Dadou et al., 2020b) were adopted in this study for the manufacture of HCTZ-RMP based FDC using HME.

\subsection{HPLC analysis of RMP and HCTZ}

Having a well-established offline analytical technique is essential for the development of a robust and reliable Raman spectroscopic method for the inline quantification of APIs (Harting and Kleinebudde, 2018). Therefore, HCTZ and RMP were first analysed using offline HPLC as a reference method. The HPLC method developed in our previous work was utilised in this study (Andrews et al., 2019a). Initially, drugs were dissolved and analysed separately to distinguish the retention time of each drug, and then a mixture of drugs in solution was analysed to ensure that both drugs can be detected (data not shown). A calibration curve was constructed for $\mathrm{HCTZ}$ with a concentration range of $5-100 \mu \mathrm{g} / \mathrm{mL}$. HCTZ was detected at retention time of $1.91 \pm 0.02$ min. Linearity was observed with a goodness of fit $\left(\mathrm{R}^{2}\right)$ of 0.999 . For RMP, a concentration range of $5-50 \mu \mathrm{g} / \mathrm{mL}$ was used to establish the calibration curve. Retention time was observed at $4.33 \pm 0.04 \mathrm{~min}$. The calibration curve showed a goodness of fit $\left(\mathrm{R}^{2}\right)$ of 0.997 . The developed HPLC method was able to detect the two drugs (HCTZ and RMP) 
simultaneously in the FDC extrudates. Well-resolved peaks associated with pure HCTZ and RMP were observed (Figure S1).

\subsection{Offline Raman spectroscopy}

Raman spectroscopy was used in this work as a PAT tool due to its high capability to analyse drugs in a rapid, non-invasive manner using a wide range of physical forms of samples (Dadou et al., 2020a). Moreover, a Raman probe can be conveniently installed in the extruder die, allowing for inline measurements (Andrews et al., 2019a). In order to assess the feasibility of using inline Raman spectroscopy to distinctively quantify RMP and HCTZ, it was important to examine if any of the specific Raman shifts assigned to characteristic groups of each drug can be unequivocally captured in the spectra. Initially, offline Raman spectra were obtained for raw drugs (RMP and HCTZ) and a physical mixture of blank formulation (polymers and plasticiser) to identify the characteristic shifts and wavenumber regions for each drug showing no overlaps with other FDC components (Figure 1). Raman shifts representing characteristic groups of RMP molecule were observed at $1004 \mathrm{~cm}^{-1}$ and $1654 \mathrm{~cm}^{-1}$. The Raman region surrounding the $1004 \mathrm{~cm}^{-1}$ shift, assigned to the aromatic ring, was chosen to quantify RMP in this study since it was the most intense peak and showed no overlapping with other Raman shifts obtained for HCTZ and/or excipients included in the formulation. Additionally, a wavenumber region that includes this shift $\left(950-1250 \mathrm{~cm}^{-1}\right)$ was successfully employed in our previous studies to quantify RMP during HME.

For HCTZ, the most intense shift was observed at $265 \mathrm{~cm}^{-1}$. In addition, a well resolved peak was detected at $709 \mathrm{~cm}^{-1}$. Therefore, the wavenumber regions surrounding the two peaks (200400 and $630-730 \mathrm{~cm}^{-1}$ ) were considered to select the region(s) that better corresponds to HCTZ content in the spectra collected during HME.

\subsection{Optimisation of Wavenumber Region Selection from Inline Raman Spectra}


Spectral pre-treatment is a vital tool in chemometrics in order to eliminate random noise and to reduce baseline drift in the signal, due to the high pressure and temperature in the extruder (Dadou et al., 2020b). Additionally, spectral pre-treatment improves the accuracy and precision of chemometrics based methods of quantification by enhancing spectral information (Eriksson et al., 2013). Standard normalised variate (SNV) and first derivative (1D) spectral pre-treatment filters were considered and applied on the inline Raman spectra collected during extrusion. For both filters, data were further processed using multiplicative scatter correction (MSC) and Savitzky-Golay (SG) filters. Raman spectra obtained offline for HCTZ, RMP and blank formulation following pre-treatment are shown in Figure $1(b, c)$. It is evident that the same distinctive regions of each drug in raw spectra are still observed in the pre-treated spectra, thus can be employed for the optimisation.

Principal component analysis (PCA) was performed to aid in selecting appropriate spectral filters and wavenumber regions that can extract the information associated with the response (drug content in this case) needed to establish the inline Raman based calibration models. PCA is utilized to visualise the partition in the dataset collected for samples (observations) and try to find pattern/categories based on latent variables known as Principal Components (PCs) (Pauli et al., 2019). In this work, Raman spectra obtained from the calibration set during the HME process were used as the dataset (x-variables), whereas the concentrations of the drugs (HCTZ and RMP) were assigned as the response (y-variables). PCA was performed for each drug individually to gain an overview on the distribution of the observations and optimise the wavenumber region that can capture the variation in the spectra associated with drug content. For HCTZ, PCA was initially performed on the wavenumber region that showed the most intense Raman shifts (200-400 $\mathrm{cm}^{-1}$ ), namely Region 1 . It can be observed from Figure 2 that the intensity of the peaks in Region 1 are directly proportional to the concentration of HCTZ in the calibration formulations. This was valid for both SNV and 1D spectra. PCA scatterplots (Figure 3) showed that the main scores of the observations are oriented along the first principal 
component (PC1) with the highest PC1 score corresponded to the extrudates containing $40 \%$ w/w of HCTZ (blue) while a low value of PC1 was associated with HCTZ concentration of $6.5 \% \mathrm{w} / \mathrm{w}$ (orange). It is evident that the data is following a simple trend where observations are oriented along PC1 axis with HCTZ concentration. However, the partition of the data is poor. It can be remarked that SNV spectra of higher concentrations of HCTZ occupied narrow space in the scatterplots with clear overlapping (Figure 3a). Although better data partition was observed at lower concentrations of HCTZ (6.5 and $12.5 \% \mathrm{w} / \mathrm{w})$, observations representing the same concentration are highly scattered. On the other hand, 1D spectra showed poor partition for all HCTZ concentrations except for the highest concentration (C5, 40\% w/w). Results obtained clearly indicate the complexity of developing a well-resolved PCA model for HCTZ using a single Raman wavenumber region which imply that Region 1 is not sufficient to describe HCTZ in the FDC extrudates.

After further investigation, the wavenumber region of $630-730 \mathrm{~cm}^{-1}$ was selected as Region 2 considering the significant variation observed in Raman signals (x-variable) with the response $(\mathrm{HCTZ} \% \mathrm{w} / \mathrm{w})$ as outlined in Figure $2 \mathrm{c}, \mathrm{d}$. Region 2 was pre-processed using the same spectral filters and was combined to Region 1 to highlight HCTZ content in the manufactured FDCs. PCA score scatterplots acquired from the two regions are shown in Figure 3 c,d. It can be observed that a better partition and clustering of the observations was achieved when the two regions were combined for both pre-processing filters compared to the corresponding spectra collected from Region 1. This was more evident when SNV was applied as the pre-processing filter (Figure 3c). PC1 was able to capture the variation in Raman spectra caused by the differences in HCTZ content in the FDC calibration formulations. The absence of off-limits points from the $95 \%$ confidence interval limits and the clear discrepancy between the spectra collected for the formulations having different HCTZ concentrations indicate a strong correlation between the Raman signals in the regions selected and the responses (HCTZ \%, w/w). Additionally, a loading plot obtained for SNV spectra (Figure 4) showed that PC1 was 
able to capture $98.6 \%$ of Raman spectral variation in Regions 1 and 2 combined. This further confirms that combining SNV spectra of the two regions (200-400 and 630-730 $\left.\mathrm{cm}^{-1}\right)$ provides a better representation of $\mathrm{HCTZ}$ content $(\% \mathrm{w} / \mathrm{w})$ in the inline Raman spectra and results in a responsive variable.

Similarly, two regions were considered for the optimisation of characteristic wavenumber region(s) for the quantification of RMP in the FDCs. Region 1 depicts the wavenumber region $950-1250 \mathrm{~cm}^{-1}$ which was optimised in our previous work. It is evident from Figure $5 \mathrm{a}, \mathrm{b}$ that Raman shifts observed in the region $950-975 \mathrm{~cm}^{-1}$ were inversely proportional to RMP content $(\% \mathrm{w} / \mathrm{w})$ in the formulation. It can be observed from Figure 1 that there is an overlap between Raman signals of RMP and HCTZ at wavenumber values higher than $1100 \mathrm{~cm}^{-1}$, and furthermore with excipients at wavenumber regions lower than $980 \mathrm{~cm}^{-1}$. This explains the inverse relation between Raman shifts in the region 950-975 $\mathrm{cm}^{-1}$ and RMP content as it represents the ratio of excipients in the formulation as it decreases when RMP concentration was increasing in the FDC. On the other hand, the intensity of Raman shifts in the region 1110$1130 \mathrm{~cm}^{-1}$ showed no correlation with RMP concentration in the calibration set. PCA scatterplots (Figure 6) further confirm of the unsuitability of Region 1 to quantify RMP in the manufactured FDCs. Data presented in Figure 6 a, b show poor partition with some off-limits observations from the $95 \%$ confidence interval. The scores obtained for formulations containing high RMP contents C4 and C5 showed better partition than formulations C1-3 when SNV pre-processing filter was applied. For 1D spectra, all observations were highly scattered regardless of RMP concentration in the calibration set. Moreover, some observations in C5 showed off-limits scattering from the $95 \%$ confidence interval.

Therefore, a narrower wavenumber region excluding the above-mentioned overlapping regions was considered $\left(980-1100 \mathrm{~cm}^{-1}\right)$ for the quantification of RMP in FDC and was assigned as Region 2. The intensity of Raman shifts in Region 2 was shown to be directly proportional to RMP concentration in calibration formulations (Figure $5 \mathrm{c}, \mathrm{d}$ ). This was valid for both SNV 
and 1D pre-processed spectra. PCA score scatterplots presented in Figure 6 (c, d) showed improved partition of the data when the selected Raman region was narrowed. The score scatter plots showed a clear distinction between the observations from different calibration mixtures. Observations having the highest PC1 score corresponded to the extrudates containing $15 \% \mathrm{w} / \mathrm{w}$ of RMP (blue) while a low value of PC1 was associated with RMP content of $2.5 \%$ w/w (red). Clusters corresponding to RMP content in formulations C1-3 were observed in the negative values of $\mathrm{PC} 1$, whereas clusters representing RMP \% w/w in $\mathrm{C} 4$ and $\mathrm{C} 5$ of were localised in the positive side of PC1. This was evident in both SNV and 1D spectra. SNV pre-processing filter was selected to establish the predictive calibration model for RMP since it was used for the development of HCTZ calibration model. Loading plot obtained for Region 2 of SNV spectra (Figure 7) showed that PC1 was able to capture $91.9 \%$ of spectral variation. This confirms that inline Raman SNV spectra of Region $2\left(980-1100 \mathrm{~cm}^{-1}\right)$ corresponded to the variation in RMP content $(\% \mathrm{w} / \mathrm{w})$ in the FDCs.

To further confirm the suitability of the selected wavenumber region(s) and pre-processing filters for the quantification of RMP and HCTZ, calibration models were established for each spectral region/filter investigated using partial least squares regression. Results obtained for performance indicators (Table S1) support the results obtained from PCA and imply the suitability of selected regions (Region 1-2 for HCTZ and Region 2 for RMP) and filter (SNV) for the purpose of measuring the quantity of drugs during HME.

\subsection{The Development and Validation of a Raman-Based PAT Method}

FDA and EMA guidance documents endorse the use of chemometrics, in particular partial least squares (PLS) predictive models, to fully realise the potential of PAT tools (FDA, 2015). In order to have accurate predictions of $\mathrm{API}(\mathrm{s})$ content during the process, reliable models offering high predictability are required (EMA, 2009). In this work, a calibration set composed of five formulations (C1-5) as outlined in Table 1 was extruded. Raman spectra were collected during the HME process. Twenty spectra were collected from each formulation. Thus, a total 
of 100 spectra ( 5 concentration levels $\times 20$ spectra per concentration) comprising $3226 \times 100$ data points were used to establish the Raman based calibration models. The actual concentration of HCTZ and RMP in the manufactured FDCs were measured post-extrusion using offline HPLC as a reference method of analysis. PLS models were established by regressing the dataset obtained from inline Raman measurements against the actual concentration of each drug as measured using HPLC. For HCTZ, a PLS calibration model was developed using the SNV pre-processed spectra for Raman Regions 1 and 2 (200-400 and 630$730 \mathrm{~cm}^{-1}$, respectively). SNV pre-processing filter was selected to establish the predictive PLS model for RMP using Raman Region $2\left(980-1100 \mathrm{~cm}^{-1}\right)$. Developed PLS models are presented in Figure 8.

To ensure the best fit of regressed data is achieved, different numbers (2-5) of principal components (PCs) were considered for each calibration model (Table 2). Metrics used to evaluate models performance when varying the number of PCs were the residual errors of estimation (RMSEE) and cross-validation (RMSEcv) of the calibration models in addition to residual errors of prediction (RMSEP) of the validation set. Large differences between RMSEE and RMSEcv denotes poor accuracy of the calibration model. In addition, high values of RMSEP indicates poor predictability of the models and signifies the need for further optimisation. Since RMSEP indicates the predictability of the models based on an external validation set, it is considered as the most relevant error parameter when developing a model and will be used to decide the number of PCs to include in the PLS model of each drug. For the development of a PLS calibration model for HCTZ, two PCs were not sufficient to quantify HCTZ accurately. Reported values for RMSEE and RMSEcv exceeded 2\%. No significant differences were observed in the values obtained for coefficients of determinations $\left(\mathrm{R}^{2} \mathrm{X}\right.$ and $\mathrm{R}^{2} \mathrm{Y}$ ) and model validity $\left(\mathrm{Q}^{2}\right)$ when 3 - 5 PCs were used. Four PCs were chosen for HCTZ calibration model, since the difference between the values reported for RMSEE and RMSEcV was low and, more importantly, RMSEP value was minimal indicating more accuracy in 
predicting HCTZ concentration in the unknowns. Similarly, the PLS model of RMP was established using three PCs, since the gap between RMSEE and RMSEcv terms and the value of RMSEP were increasing when more PCs were added implying a drop in model accuracy (Table 2).

Specificity, which depicts the ability of the method to identify the analyte of interest from other components in the formulation, was evaluated by comparing the original spectra collected offline for drugs and excipients. As shown in Figure 1, there is no overlap in the Raman regions selected for each drug and the second drug or the excipients, used to formulate the FDCs. Furthermore, loading spectra obtained from PLS models developed for HCTZ (Figure 4) and RMP (Figure 7) confirmed the selectivity of the models developed as such PC1 was able to capture $98.6 \%$ and $91.9 \%$ of the variation in the spectra associated with the change in HCTZ and RMP concentrations in the calibration set, respectively.

Linearity was estimated from the established PLS calibration models for each drug. The predicted values of HCTZ and RMP from Raman spectra were plotted, separately, against the corresponding measured values using the reference HPLC method (Figure 8). The PLS calibration models showed good correlation between the measured/true and predicted concentrations of the drugs in the calibration set. Coefficient of determination $\left(\mathrm{R}^{2}\right)$, slope and intercept were the metrics used to assess linearity. Results obtained (Table 3) clearly indicate excellent linearity for both models. The slope values were 1 whereas the values of the intercept were very close to zero. The regressed lines were able to fit 98.6 and $97.4 \%$ of the observations associated with HCTZ and RMP models, respectively.

The accuracy of a method of analysis is reflected in the agreement between the actual and the predicted values (ICH, 2005). Herein, accuracy was reported as RMSEE, RMSEcv and RMSEP for each model. RMSEE and RMSEcv were calculated using the calibration set whereas the validation set was used to calculate RMSEP. The values calculated for RMSEE, RMSEcv and RMSEP from each model are reported in Table 3. Small differences between 
RMSEE and RMSEcv were observed indicating high accuracy of both models. Similarly, an excellent predictability was evident for the two models as RMSEP values calculated for HCTZ and RMP in the validation set were 1.237 and $1.007 \%$, respectively. This indicates the ability of the developed models to predict unknowns accurately.

\subsection{Evaluation of the predictive performance of the developed PLS based PAT models}

To evaluate the predictive performance and precision of the developed PLS calibration models, Raman spectra were collected inline from a validation set composed of FDCs manufactured at concentration levels of HCTZ and RMP different from the calibration set (V1-3, Table 1). Raman spectra were acquired at predetermined time intervals during the extrusion process and samples of produced extrudates, that match the time at which the spectra were obtained, were collected and analysed for drugs content. The prediction performance of the developed PLS calibration models were assessed by reporting RMSEP values for the drugs at each time point. Additionally, relative bias was estimated for the drugs at each concentration level to quantify the deviation from the actual values measured using HPLC. A deviation of $\pm 10 \%$ from the true value was deemed acceptable (Saerens et al., 2014). On the other hand, relative standard deviation (RSD) was calculated to evaluate precision by measuring the variance between the predicted concentration of the drugs from triplicate measurements at each time point for the same sample. The PLS developed models for both drugs showed high precision and predictability of drugs concentration in the FDCs manufactured (Table 4). Moreover, PCA scatterplot generated from the Raman spectra obtained inline for formulations V1-3 (Figure 9) shows good partition of the data in the plane. The main scores of the observations are oriented along PC1 with drug concentration (for both drugs) which confirms the capability of the developed models to capture the variation in the spectra associated with drug concentration in the validation set. Calculated relative biases and RSD values were below $10 \%$ for all samples (Table 4). However, it was observed that the values become lower when higher concentration of drug is included in the validation set. Relative biases reported for RMP and HCTZ in V1 
were 27 and 10 times higher than V3. Additionally, RSD values obtained from V1 were almost twice the values of V3 for both drugs. The observed drop in relative biases values in V2 and V3 can be explained by the well-known weak scatter effect of Raman; limiting its sensitivity for lower concentrations of analysts. Nevertheless, reported values of relative bias were within the accepted limit $(\leq 10 \%)$.

A paired t-test was considered to further investigate the predictive performance of the PLS models and outline any significant difference in the predicted concentrations of the drugs in the validation set when correlated to the true values measured using HPLC. The difference was considered significant at p-values lower than 0.05 . Results reported in Table 5 revealed that there were no significant differences between the drugs content measured using HPLC and predicted from the established PLS models $(\mathrm{p}>0.05)$. Results obtained reveal the excellent predictability of the developed PAT methods and their high accuracy in predicting the concentration of HCTZ and RMP in the FDCs. This further confirms the high potential of inline Raman spectroscopy and its suitability as an in-process method of quantification to monitor the quality of the product and/or process. 


\section{Conclusion}

The feasibility of quantifying two drugs (HCTZ and RMP) during the hot melt extrusion of fixed dose combinations was confirmed in this study. Results obtained indicate that a careful selection of the spectral range that can identify each drug unequivocally in the FDC is of paramount importance for the development of reliable PAT predictive models. Raman spectra collected inline for a HCTZ-RMP based FDC were converted into principle components maps with the aid of chemometrics. When suitable Raman regions were selected and proper preprocessing spectral filters were applied, variations in the spectra associated with the levels of HCTZ and RMP in the FDC were successfully captured and mapped into clusters. Reliable calibration models were established across therapeutically relevant dosages of the drugs using PLS regression. Furthermore, validation requirements in accordance to current international standards were met. Reported values for the errors of estimation, cross validation and prediction were low confirming the high accuracy of the developed PLS models in predicting drugs concentrations with acceptable deviation from true values, which were verified by offline HPLC measurements. The output of this study allows in-process quality control of multiple drugs, facilitating the integration of continuous processing platforms as means of manufacture of FDCs in the pharmaceutical industry. Furthermore, it allows the implementation of PAT tools as routine methods of analysis within the laboratory with a high potential of replacing the current conventional, destructive offline methods. 


\section{References}

Abegaz, T.M., Shehab, A., Gebreyohannes, E.A., Bhagavathula, A.S., Elnour, A.A., 2017. Nonadherence to antihypertensive drugs a systematic review and meta-analysis. Medicine (Baltimore). 96. https://doi.org/10.1097/MD.0000000000005641

Andrews, G.P., Jones, D.S., Senta-Loys, Z., Almajaan, A., Li, S., Chevallier, O., Elliot, C., Healy, A.M., Kelleher, J.F., Madi, A.M., Gilvary, G.C., Tian, Y., 2019a. The development of an inline Raman spectroscopic analysis method as a quality control tool for hot melt extruded ramipril fixed-dose combination products. Int. J. Pharm. 566, 476-487. https://doi.org/10.1016/j.ijpharm.2019.05.029

Andrews, G.P., Li, S., Almajaan, A., Yu, T., Martini, L., Healy, A., Jones, D.S., 2019b. Fixed Dose Combination Formulations: Multilayered Platforms Designed for the Management of Cardiovascular Disease. Mol. Pharm. 16, 1827-1838. https://doi.org/10.1021/acs.molpharmaceut.8b01068

Dadou, S.M., El-Barghouthi, M.I., Antonijevic, M.D., Chowdhry, B.Z., Badwan, A.A., 2020a. Elucidation of the Controlled-Release Behavior of Metoprolol Succinate from Directly Compressed Xanthan Gum/Chitosan Polymers: Computational and Experimental Studies. ACS Biomater. Sci. Eng. 6, 21-37. https://doi.org/10.1021/acsbiomaterials.8b01028

Dadou, S.M., Senta-Loys, Z., Almajaan, A., Li, S., Jones, D.S., Healy, A.M., Tian, Y., Andrews, G.P., 2020b. The development and validation of a quality by design based process analytical tool for the inline quantification of Ramipril during hot-melt extrusion. Int. J. Pharm. 584, 119382. https://doi.org/10.1016/j.ijpharm.2020.119382

Dierickx, L., Saerens, L., Almeida, A., De Beer, T., Remon, J.P., Vervaet, C., 2012. Coextrusion as manufacturing technique for fixed-dose combination mini-matrices. Eur. J. Pharm. Biopharm. 81, 683-689. https://doi.org/10.1016/j.ejpb.2012.03.018

EMA, 2017. Guideline on Clinical Development of Fixed Combination Medicinal Products. Eur. Med. Agency. EMA/CHMP/158268/2017.

EMA, 2009. Guideline on the use of near infrared spectroscopy by the pharmaceutical industry and the data requirements for new submissions and variations.

Eriksson, L., Byrne, T., Johansson, E., Trygg, J., Vikström, C., 2013. Multi- and Megavariate Data Analysis Basic Principles and Applications, Umetrics Academy. https://doi.org/10.1198/tech.2003.s162

FDA, 2015. Development and Submission of Near Infrared Analytical Procedures Guidance for Industry. Cent. Drug Eval. Res.

FDA, 2004. Guidance for Industry: PAT - a framework for innovative pharmaceutical 
development, manufacturing, and quality assurance. Rockville, MD.

Hao, J., Rodriguez-Monguio, R., Seoane-Vazquez, E., 2015. Fixed-dose combination drug approvals, patents and market exclusivities compared to single active ingredient pharmaceuticals. PLoS One 10. https://doi.org/10.1371/journal.pone.0140708

Harting, J., Kleinebudde, P., 2018. Development of an in-line Raman spectroscopic method for continuous API quantification during twin-screw wet granulation. Eur. J. Pharm. Biopharm. 125, 169-181. https://doi.org/10.1016/j.ejpb.2018.01.015

Hisada, H., Okayama, A., Hoshino, T., Carriere, J., Koide, T., Yamamoto, Y., Fukami, T., 2020. Determining the Distribution of Active Pharmaceutical Ingredients in Combination Tablets Using Near IR and Low-Frequency Raman Spectroscopy Imaging. Chem. Pharm. Bull. (Tokyo). 68, 155-160. https://doi.org/10.1248/cpb.c19-00791

ICH, 2005. Harmonised Tripartite Guideline. Validation of Analytical Procedures: Text and Methodology Q2 (R1). Website: http://www.ich.org/cache/compo/363-272-1.html.

Kelleher, J.F., Gilvary, G.C., Madi, A.M., Jones, D.S., Li, S., Tian, Y., Almajaan, A., SentaLoys, Z., Andrews, G.P., Healy, A.M., 2018. A comparative study between hot-melt extrusion and spray-drying for the manufacture of anti-hypertension compatible monolithic fixed-dose combination products. Int. J. Pharm. 545, 183-196. https://doi.org/10.1016/j.ijpharm.2018.05.008

Kishore, S.P., Salam, A., Rodgers, A., Jaffe, M.G., Frieden, T., 2018. Fixed-dose combinations for hypertension. Lancet 392, 819-820. https://doi.org/10.1016/S0140-6736(18)31814-2

Koide, T., Takeuchi, Y., Otaki, T., Yamamoto, K., Shimamura, R., Ohashi, R., Inoue, M., Fukami, T., Izutsu, K. ichi, 2020. Quantification of a cocrystal and its dissociated compounds in solid dosage form using transmission Raman spectroscopy. J. Pharm. Biomed. Anal. 177, 112886. https://doi.org/10.1016/j.jpba.2019.112886

Lee, S.L., 2017. Modernizing the way drugs are made: A transition to continuous manufacturing [WWW Document]. US Food Drug Adm. https://doi.org/10.1002/cncr.23522

Nomura, K., Titapiwatanakun, V., Hisada, H., Koide, T., Fukami, T., 2020. In situ monitoring of the crystalline state of active pharmaceutical ingredients during high-shear wet granulation using a low-frequency Raman probe. Eur. J. Pharm. Biopharm. 147, 1-9. https://doi.org/10.1016/j.ejpb.2019.12.004

Oo, C., Sy, S.K.B., 2018. Fixed-dose combinations: a potential means to boost drug development for selected drugs. Drug Discov. Today 23, 457. https://doi.org/10.1016/j.drudis.2017.11.001 
Pauli, V., Roggo, Y., Pellegatti, L., Nguyen Trung, N.Q., Elbaz, F., Ensslin, S., Kleinebudde, P., Krumme, M., 2019. Process analytical technology for continuous manufacturing tableting processing: A case study. J. Pharm. Biomed. Anal. 162, 101-111. https://doi.org/10.1016/j.jpba.2018.09.016

Saerens, L., Segher, N., Vervaet, C., Remon, J.P., De Beer, T., 2014. Validation of an in-line Raman spectroscopic method for continuous active pharmaceutical ingredient quantification during pharmaceutical hot-melt extrusion. Anal. Chim. Acta 806, 180-187. https://doi.org/10.1016/j.aca.2013.11.020

Salam, A., Kanukula, R., Esam, H., Bahiru, E., Sharma, A., Heller, D., Huffman, M., Vedanthan, R., Agarwal, A., Jaffe, M.G., 2019. An application to include blood pressure lowering drug fixed dose combinations to the model list of essential medicines lists for the treatment of essential hypertension in adults.

Tiwari, R. V., Patil, H., Repka, M.A., 2016. Contribution of hot-melt extrusion technology to advance drug delivery in the 21st century. Expert Opin. Drug Deliv. 13, 451-464. https://doi.org/10.1517/17425247.2016.1126246

US Food and Drug Administration, 2014. Guidance for industry: new chemical entity exclusivity determinations for certain fixed-combination drug products. Rockville, Maryl. US Dep. Heal. Hum. Serv.

Wilson, M., Williams, M.A., Jones, D.S., Andrews, G.P., 2012. Hot-melt extrusion technology and pharmaceutical application. Ther. Deliv. 3, 787-797. https://doi.org/10.4155/tde.12.26

World Health Organization, 2019. Hypertenstion [WWW Document]. URL https://www.who.int/news-room/fact-sheets/detail/hypertension (accessed 6.9.20). 


Figure 1. Raman spectra collected offline for pure drugs (HCTZ and RMP) and physical mixture of excipients only (Blank). Data represent: (a) raw, (b) SNV, and (c) 1D spectra. 

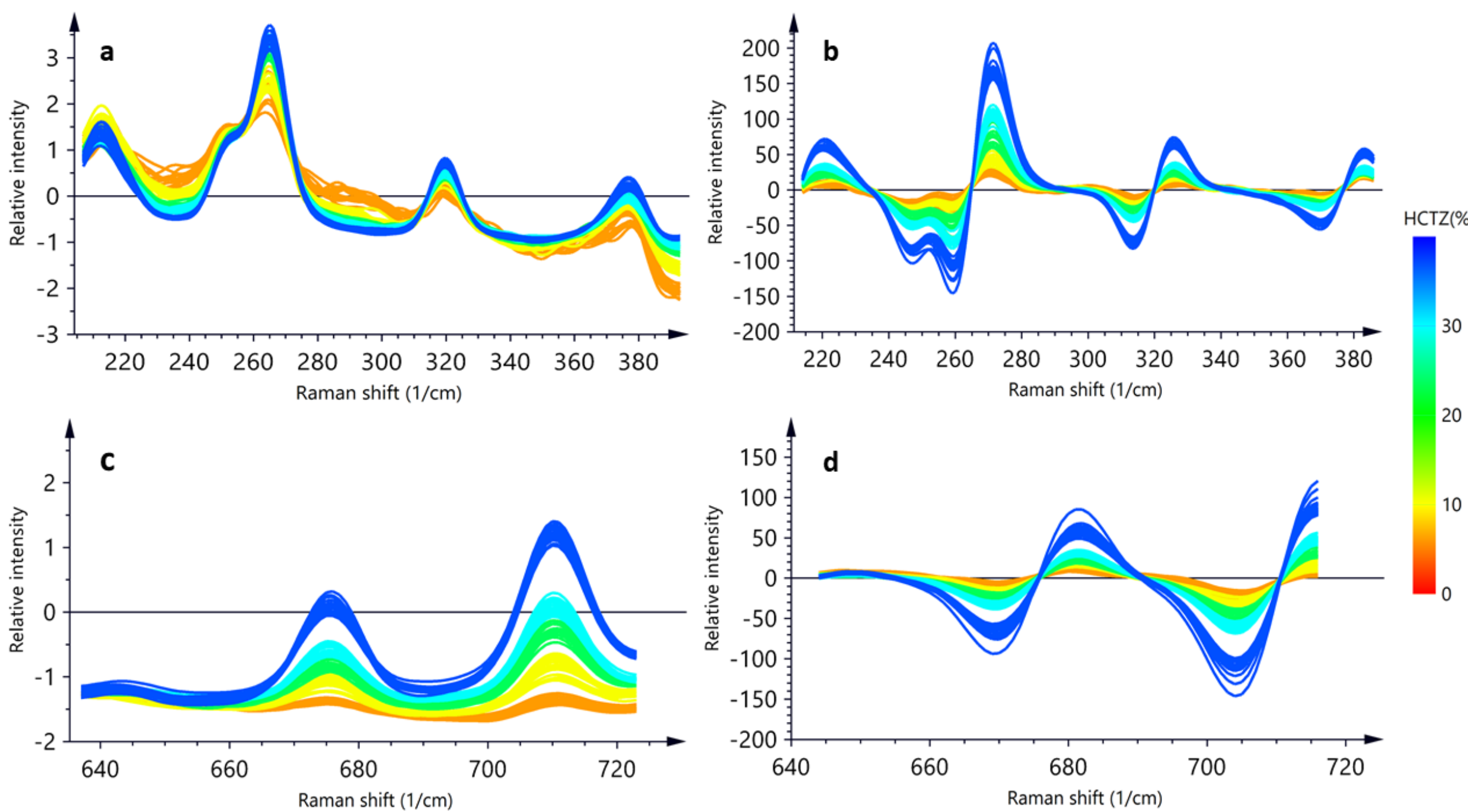

Figure 2. Changes in the intensity of Raman shifts with the concentration of HCTZ at: (a) Region 1 using SNV filter, (b) Region 1 using 1D filter, (c) Region 2 using SNV filter, and (d) Region 2 using 1D filter. Colour code on the right hand side denotes HCTZ concentration (\% w/w) in the calibration set. Region $1\left(200-400 \mathrm{~cm}^{-1}\right)$ and Region $2\left(630-730 \mathrm{~cm}^{-1}\right)$. 

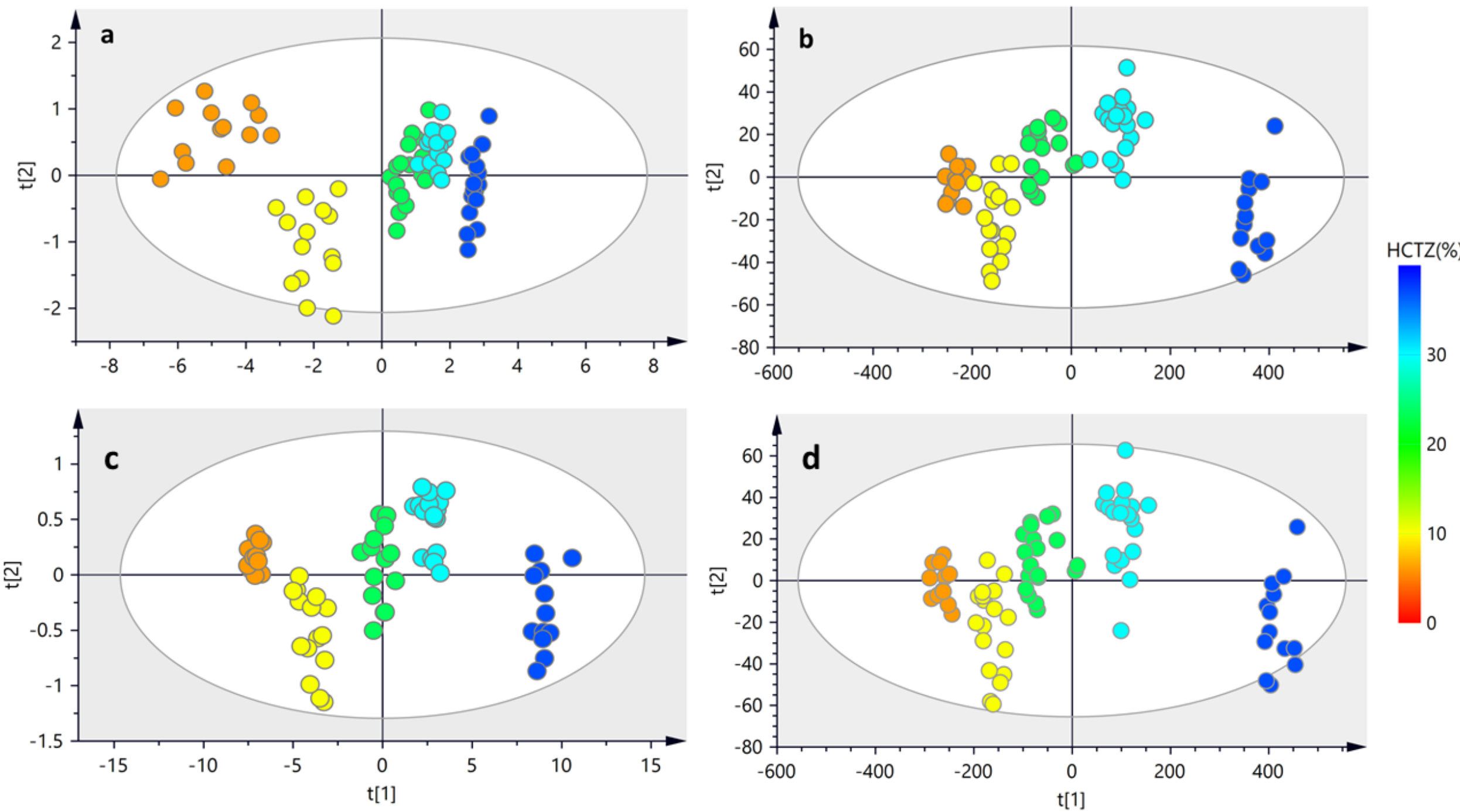

Figure 3. PCA score scatterplots generated from the inline Raman spectra collected for HCTZ at: (a) Region 1 using SNV filter, (b) Region 1 using $1 D$ filter, (c) Regions 1-2 using SNV filter, and (d) Regions 1-2 using 1D filter. Colour code on the right side represents HCTZ concentration (\% w/w) in the calibration set (C1, orange; C2, yellow; C3, green; C4, cyan; C5, blue). Region $1\left(200-400 \mathrm{~cm}^{-1}\right)$ and Region $2\left(630-730 \mathrm{~cm}^{-1}\right)$. 


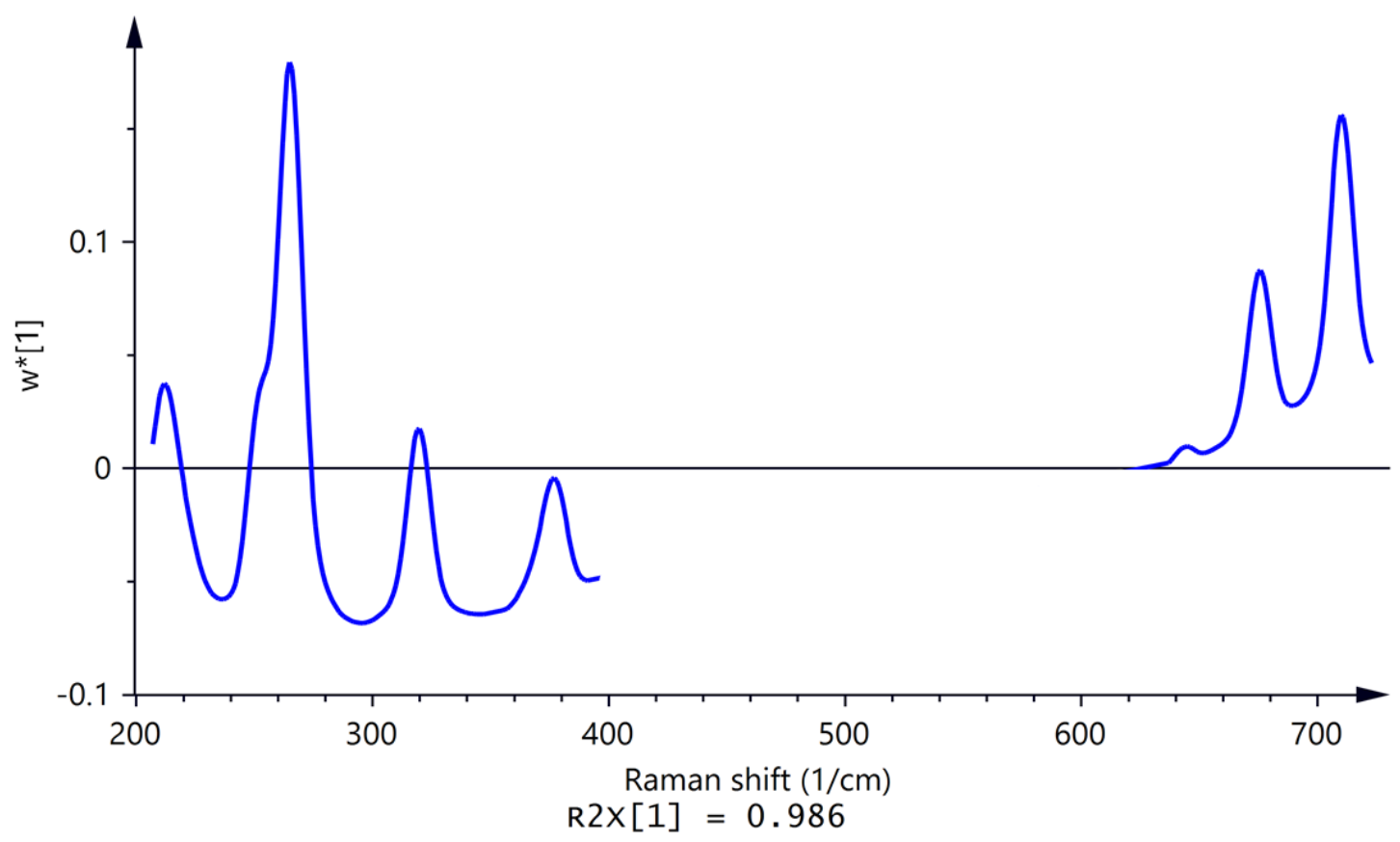

Figure 4. Loading plot of the first principal component obtained from the SNV Raman based PLS calibration model developed for HCTZ using Regions 1 and 2 combined. 

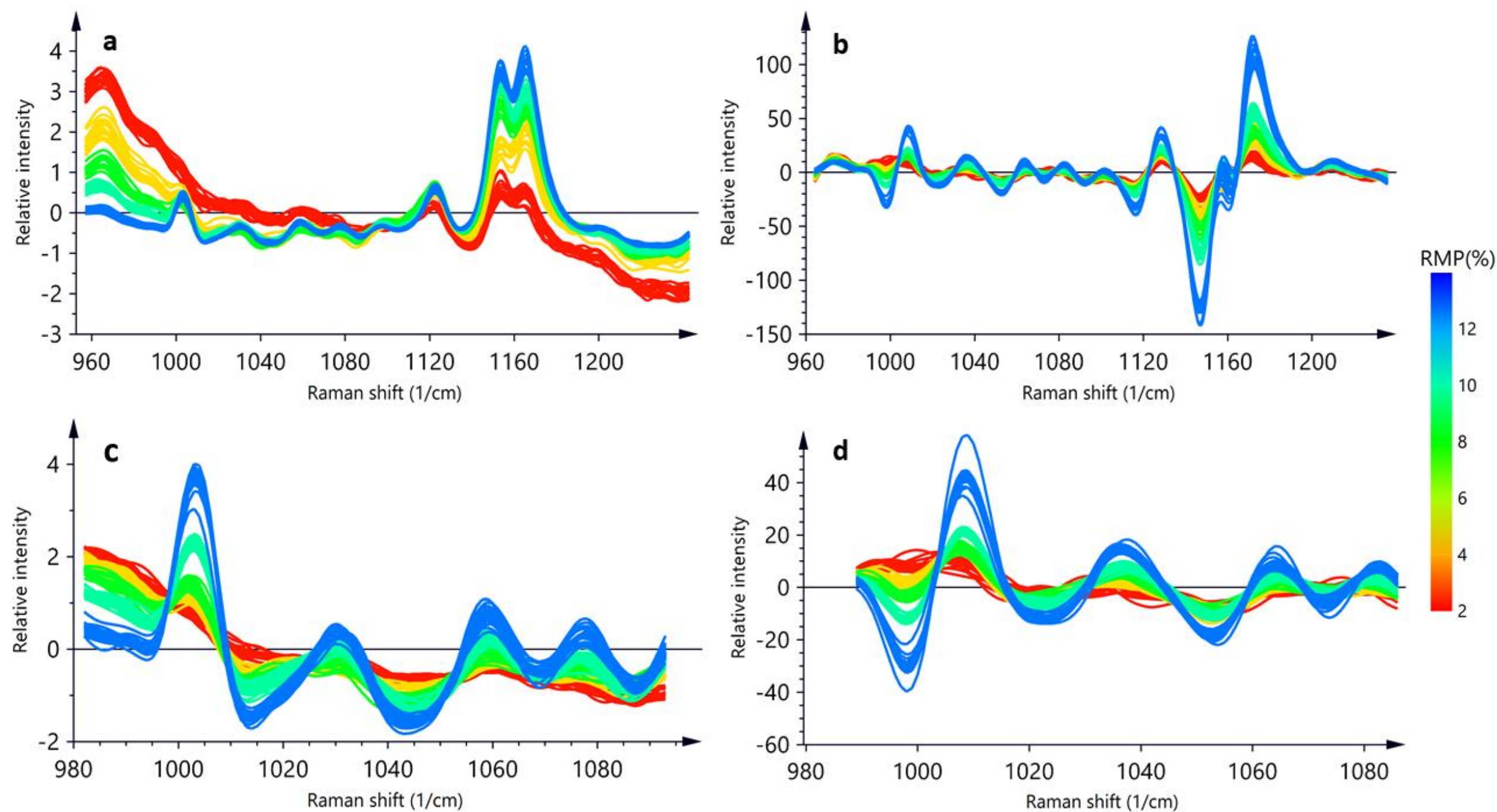

Figure 5. Changes in the intensity of Raman shifts with the concentration of RMP at: (a) Region 1 using SNV filter, (b) Region 1 using 1D filter, (c) Region 2 using SNV filter, and (d) Region 2 using 1D filter. Colour code on the right side denotes RMP concentration (\% w/w) in the calibration set of formulations. Region $1\left(950-1250 \mathrm{~cm}^{-1}\right)$ and Region $2\left(980-1100 \mathrm{~cm}^{-1}\right)$. 

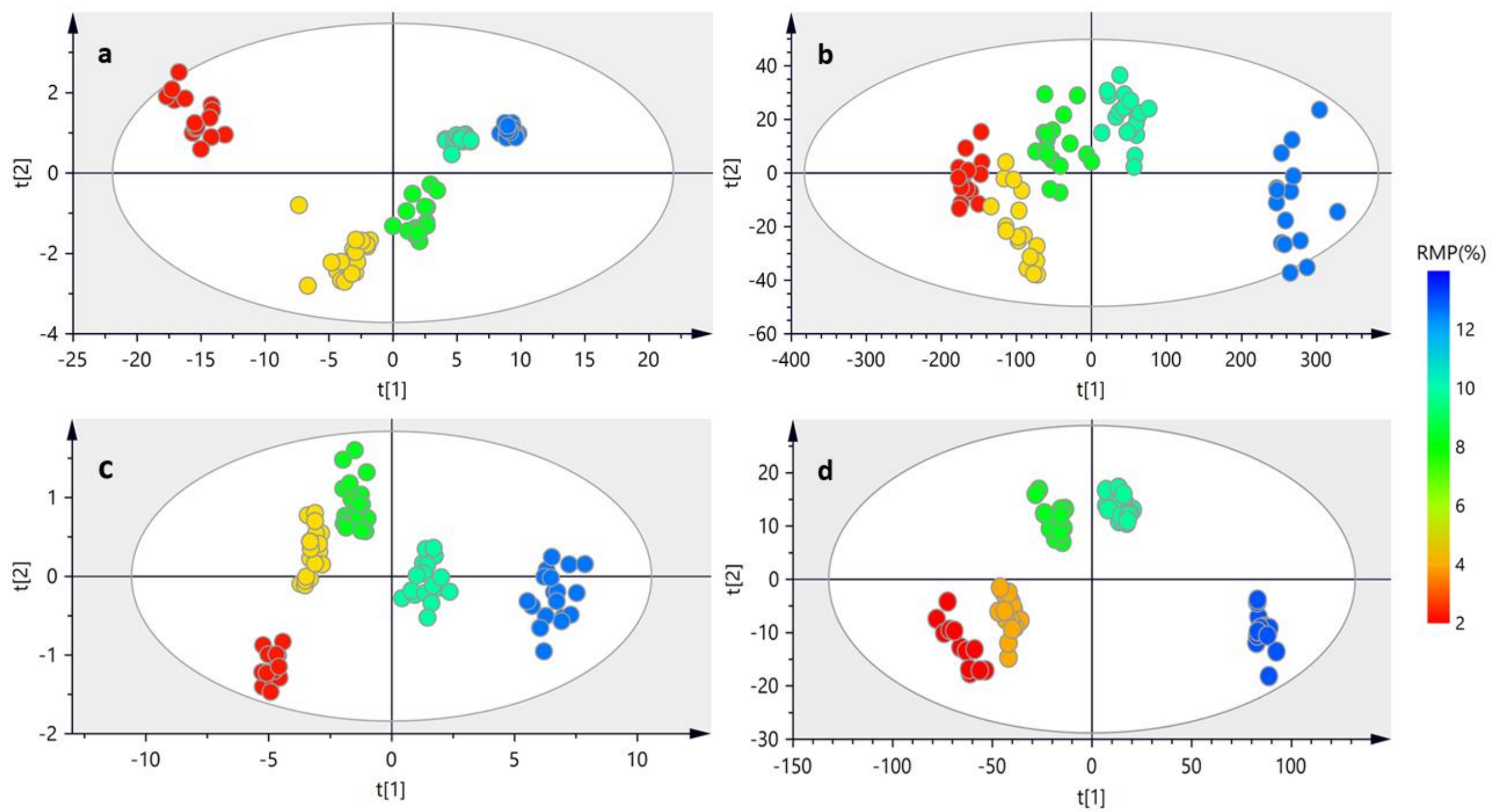

Figure 6. PCA score scatterplots generated from the inline Raman spectra collected for RMP at: (a) Region 1 using SNV filter, (b) Region 1 using 1D filter, (c) Regions 2 using SNV filter, and (d) Regions 2 using 1D filter. Colour code on the right side represents RMP concentration (\% w/w) in the calibration set formulations (C1, red; C2, yellow; C3, green; C4, cyan; C5, blue). Region $1\left(950-1250 \mathrm{~cm}^{-1}\right)$ and Region $2\left(980-1100 \mathrm{~cm}^{-1}\right)$. 


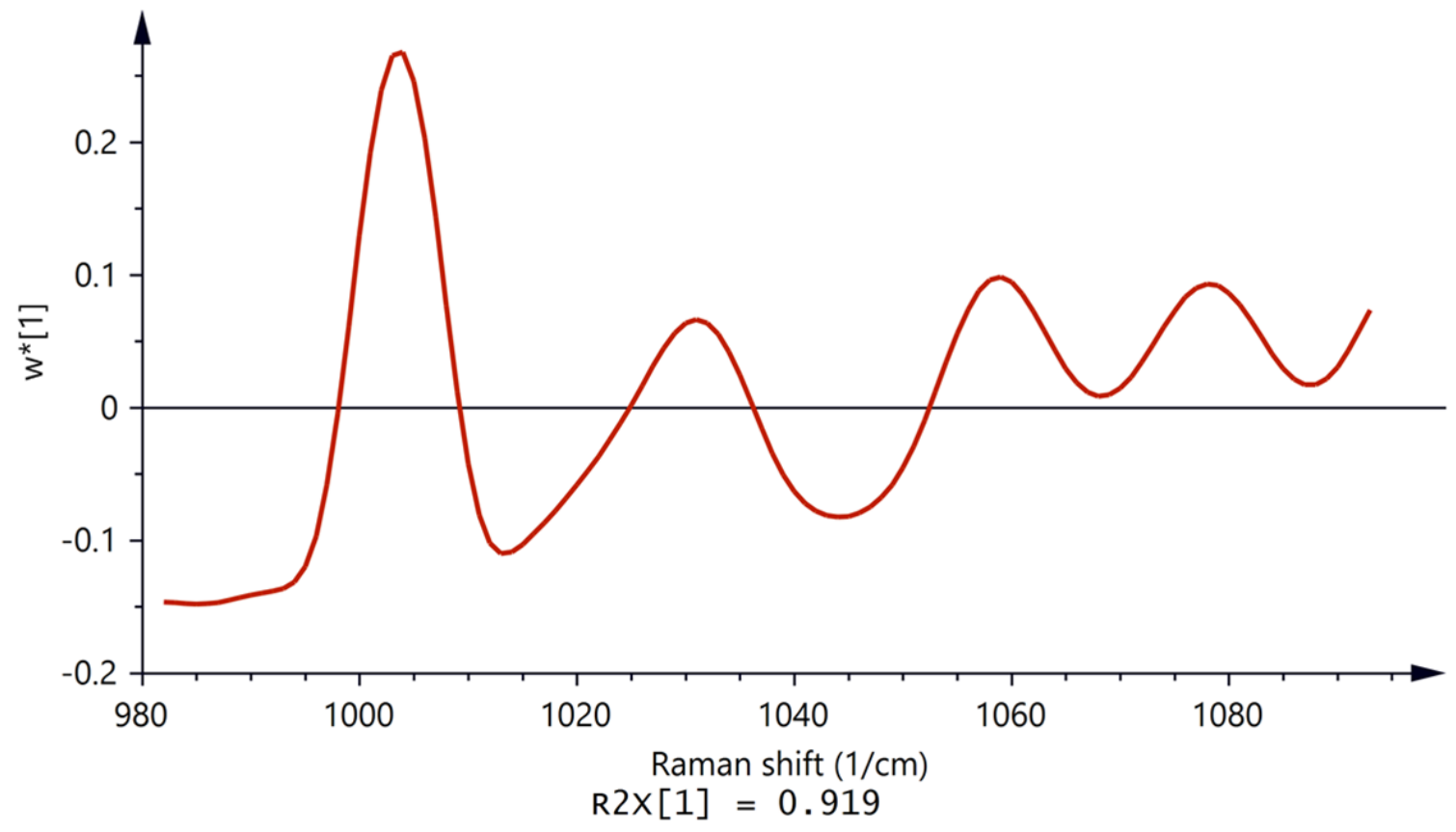

Figure 7. Loading plot of the first principal component obtained from the SNV Raman based PLS calibration model developed for RMP using Region 2. 

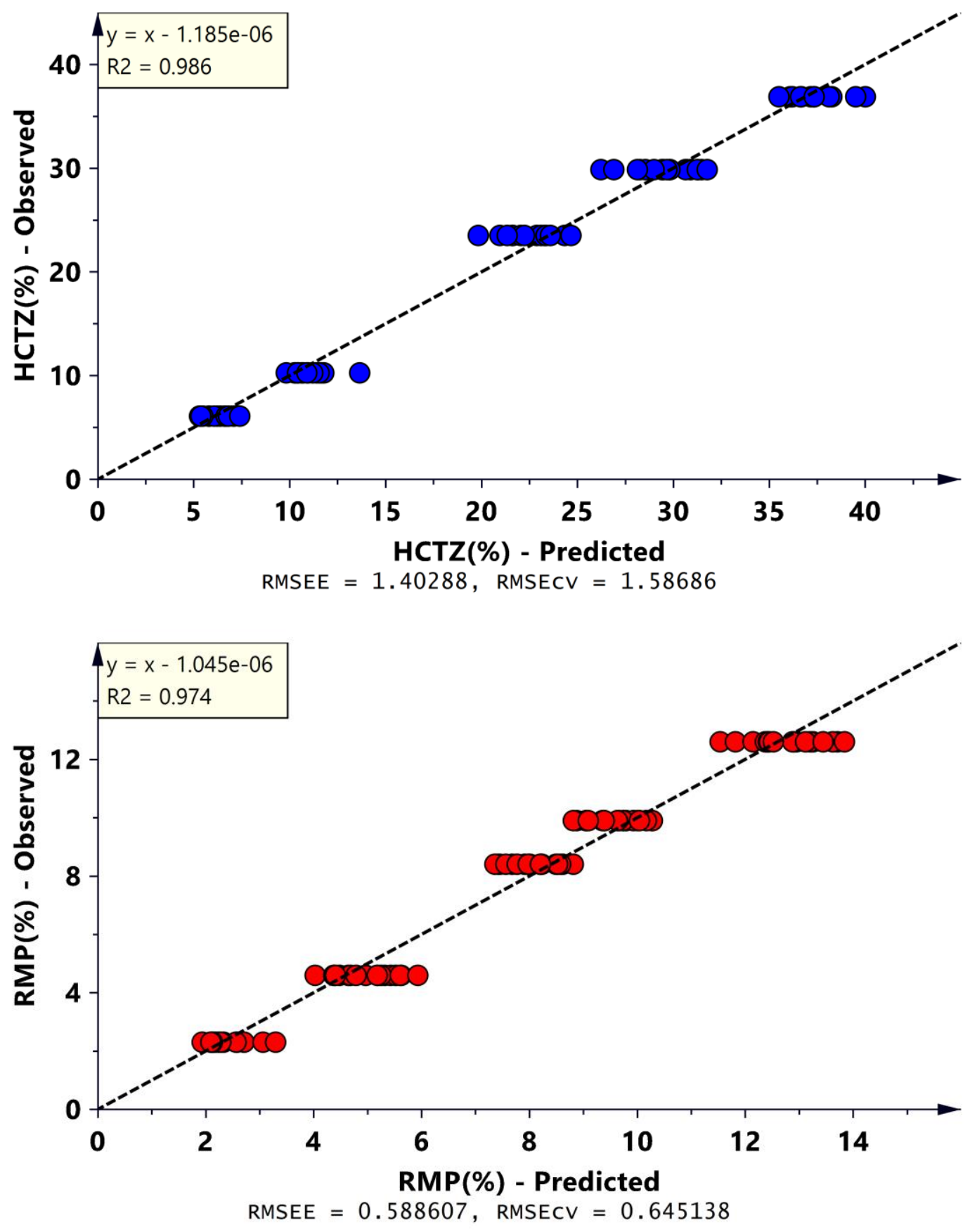

Figure 8. The developed Raman based PLS calibration models of HCTZ (blue) and RMP (red). 

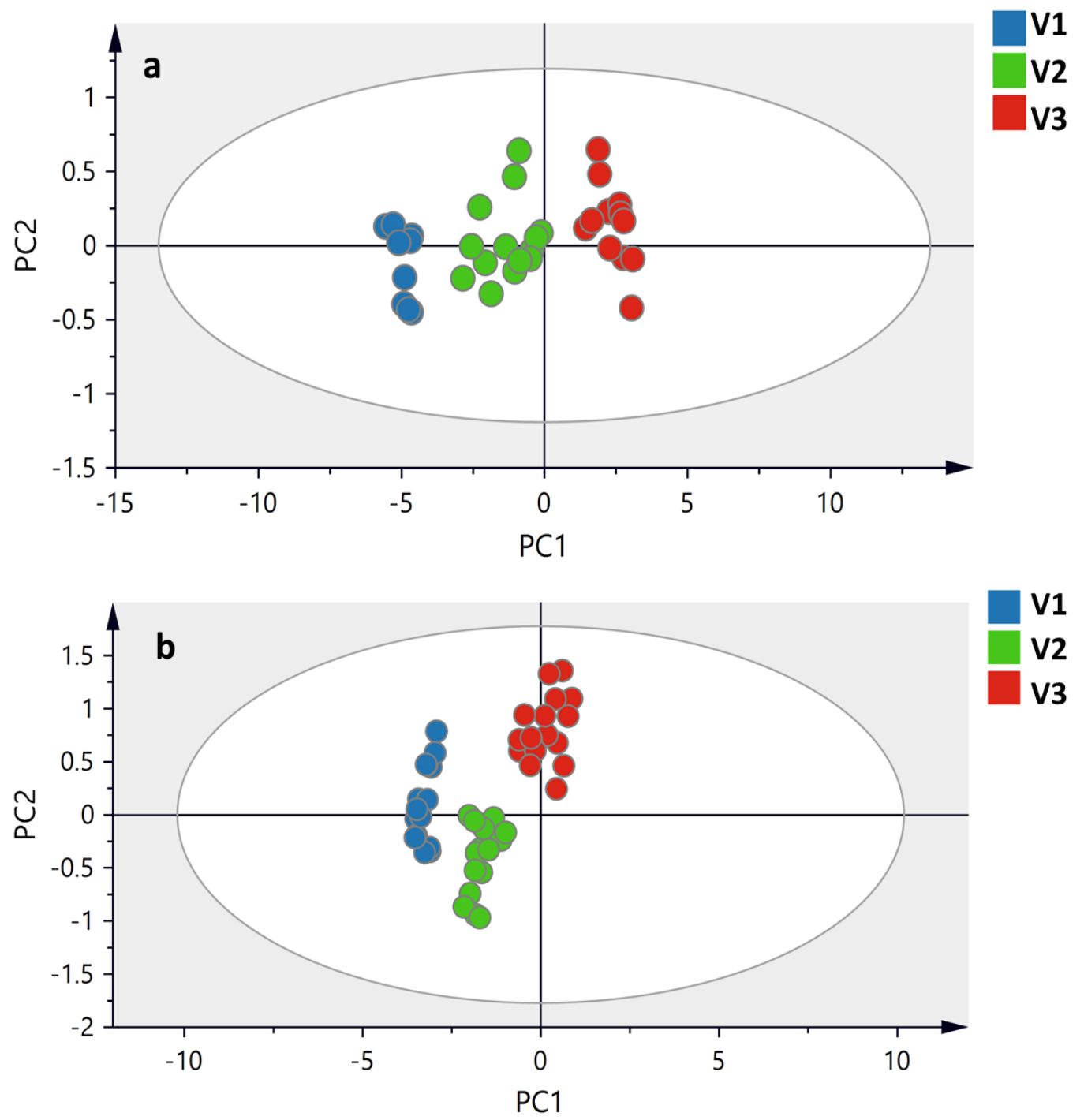

Figure 9. PCA score scatterplots obtained for the validation set using the PLS calibration models developed for the quantification of: (a) HCTZ and (b) RMP. 
Table 1. Formulations used to develop and validate Raman based PLS calibration models ${ }^{\mathrm{a}}$

\begin{tabular}{llllll}
\hline Formulation $^{\mathbf{b}}$ & HCTZ & RMP & EPO & L100 & TEC \\
\hline C1 & 6.5 & 2.5 & 41.75 & 41.75 & 7.5 \\
C2 & 12.5 & 5 & 37.5 & 37.5 & 7.5 \\
C3 & 25 & 10 & 21.25 & 21.25 & 7.5 \\
C4 & 31.5 & 12.5 & 25.75 & 25.75 & 7.5 \\
C5 & 40 & 15 & 18.75 & 18.75 & 7.5 \\
\hline V1 & 10 & 4 & 39.25 & 39.25 & 7.5 \\
V2 & 19 & 7 & 33.25 & 33.25 & 7.5 \\
V3 & 28 & 11 & 26.75 & 26.75 & 7.5 \\
\hline
\end{tabular}

${ }^{a}$ All values reported are in weight percentage $(\% \mathrm{w} / \mathrm{w})$

${ }^{b}$ Formulations C1-C5 were used as the calibration set whereas V1-V3 as the validation set 
Table 2. Model performance indicators for the HCTZ and RMP developed PLS models*

\begin{tabular}{lllllll}
\hline № of PCs & $\mathbf{R}^{\mathbf{2}}$ & $\mathbf{R}^{\mathbf{2}} \mathbf{Y}$ & $\mathbf{Q}^{\mathbf{2}}$ & $\mathbf{R M S E E}$ & $\mathbf{R M S E c v}$ & $\mathbf{R M S E P}$ \\
$\underline{\mathbf{H C T Z}}$ & & & & & & \\
$\mathbf{2}$ & 0.994 & 0.968 & 0.966 & 2.066 & 2.076 & 1.396 \\
$\mathbf{3}$ & 0.995 & 0.980 & 0.975 & 1.645 & 1.787 & 1.517 \\
$\mathbf{4}$ & 0.996 & 0.986 & 0.980 & 1.403 & 1.587 & 1.237 \\
$\mathbf{5}$ & 0.996 & 0.989 & 0.978 & 1.235 & 1.726 & 1.200 \\
\hline$\underline{\mathbf{R M P}}$ & & & & & & \\
$\mathbf{2}$ & 0.951 & 0.943 & 0.939 & 0.875 & 0.886 & 1.035 \\
$\mathbf{3}$ & 0.960 & 0.974 & 0.968 & 0.588 & 0.645 & 1.007 \\
$\mathbf{4}$ & 0.965 & 0.980 & 0.965 & 0.525 & 0.671 & 1.012 \\
$\mathbf{5}$ & 0.968 & 0.982 & 0.962 & 0.498 & 0.736 & 1.010 \\
\hline
\end{tabular}

* PCs, principal components, $R^{2} X, R^{2}$ predicted; $R^{2} Y, R^{2}$ observed; $Q^{2}$, model validity; $R M S E E$, root mean square error of estimation; RMSEcv, root mean square error of cross validation; RMSEP, root mean square error of prediction 
Table 3. Overview of validation parameters obtained for the calibration set of HCTZ and RMP using the established Raman based PLS calibration models

\begin{tabular}{lllllll}
\hline & \multicolumn{2}{l}{ Linearity } & & \multicolumn{1}{c}{ Accuracy } \\
& $\mathrm{R}^{2}$ & Intercept & Slope & RMSEE (\%) & RMSEcv (\%) & RMSEP (\%) \\
\hline HCTZ & 0.986 & $1.19 \mathrm{e}-6$ & 1 & 1.403 & 1.586 & 1.237 \\
RMP & 0.974 & $1.05 \mathrm{e}-6$ & 1 & 0.589 & 0.645 & 1.007 \\
\hline
\end{tabular}


Table 4. Intraday precision and predictability calculated for the validation set using the established PLS calibration models of HCTZ and RMP*

\begin{tabular}{lllllll}
\hline Formulation & $\underline{\text { HCTZ }}$ & & & RMP & & \\
& RSD & RMSEP & Relative bias & RSD & RMSEP & Relative bias \\
\hline V1 & 3.70 & 1.362 & 8.88 & 9.02 & 1.017 & 9.90 \\
V2 & 4.67 & 1.440 & 2.11 & 2.36 & 0.881 & 4.93 \\
V3 & 1.68 & 0.977 & 0.88 & 3.86 & 0.883 & 0.36 \\
\hline
\end{tabular}

$* R S D$, relative standard deviation; RMSEP, root mean square error of prediction. All values are percentage (\%). 
Table 5. Concentration of HCTZ and RMP in the validation set as predicted from the established PLS calibration models and measured using HPLC

\begin{tabular}{|c|c|c|c|c|c|c|}
\hline & $\underline{\text { HCTZ }}$ & & & $\underline{\mathbf{R M P}}$ & & \\
\hline Formulation & Actual* & Predicted* & p-value & Actual* & Predicted* & $\mathrm{p}$-value \\
\hline V1 & $10.19 \pm 0.40$ & $11.09 \pm 0.41$ & 0.132 & $3.79 \pm 0.18$ & $4.16 \pm 0.37$ & 0.355 \\
\hline V2 & $19.48 \pm 1.05$ & $19.06 \pm 0.89$ & 0.629 & $6.35 \pm 0.14$ & $6.03 \pm 0.14$ & 0.185 \\
\hline V3 & $27.00 \pm 2.03$ & $26.76 \pm 0.45$ & 0.865 & $9.72 \pm 1.12$ & $9.69 \pm 0.34$ & 0.960 \\
\hline
\end{tabular}

* Values reported are in weight percentage $(\% w / w)$ and represent the average $\pm S D(n=3)$. 